oral aquando da introdução da dieta sem glúten. O valor do ECOHIS total apresentou uma média de $5,2(d p=6,8)$. Verificou-se uma associação significativa entre a QdVRSO e as manifestações orais ( $\mathrm{p}<0,05)$, com exceção da hipoplasia dentária e do líquen plano oral. Conclusões: Foi relatada uma elevada percentagem de manifestações orais nas crianças/adolescentes com DC, em particular de aftas recorrentes, cárie e opacidade dentária. A QdVRSO das crianças/adolescentes com DC pode considerar-se boa, no entanto as manifestações orais associadas à DC apresentaram, de um modo geral, um elevado impacto na QdVRSO.

http://doi.org/10.24873/j.rpemd.2022.01.927

\#071 A ansiedade, medo e stress nos profissionais de saúde oral durante a pandemia de COVID-19

Ana Rita Gonçalves Rodrigo*, Inês Alexandra Costa Morais Caldas, Álvaro Amadeu Ferreira de Azevedo, Maria de Lurdes Lobo Pereira

Faculdade de Medicina Dentária da Universidade do Porto

Objetivos: Caracterizar o impacto da atual pandemia de COVID-19 na ansiedade, medo e stress nos profissionais de saúde oral. Materiais e métodos: Foi utilizado um questionário autoaplicado online realizado no Google Forms e foi enviado, via email, com o link de acesso ao questionário para três associações na área da Medicina Dentária. O questionário era composto por três partes: a primeira abordava questões relativas à caracterização sociodemográfica; na segunda foi utilizado o questionário validado de Transtorno de Ansiedade Generalizada (GAD-7); e na terceira foram colocadas questões relativas ao medo, ao stress e ao impacto que a pandemia teve na atividade profissional. Foi utilizado o programa estatístico IBM SPSS Statistics $26^{\circledR}$. As variáveis categóricas foram descritas através de frequências absolutas e relativas. As variáveis contínuas foram descritas utilizando a média e o desvio-padrão. Foi utilizado um modelo de regressão linear múltipla para testar se a ansiedade podia ser prevista pelas diferentes variáveis do questionário. Resultados: Foi obtida uma taxa de participação de 21,1\% (n=390). Verificou-se que a maioria dos participantes era do sexo feminino $(67,2 \%)$, mais de metade $(51,8 \%)$ trabalhava em clínica própria e cerca de $48,2 \%$ trabalhava na região do Norte. Através da escala GAD-7 verificou-se que $18,3 \%$ dos profissionais apresentaram uma perturbação de ansiedade moderada e grave, e 39,5\% ansiedade leve. A maioria dos participantes $(86,7 \%)$ tinha medo de infetar os familiares e amigos. Quase a totalidade dos profissionais (90\%) foi afetado negativamente na sua atividade profissional e $44,6 \%$ preocupou-se muito com a perda de rendimentos. As novas condições de trabalho afetaram o dia a dia de $42,8 \%$ dos indivíduos. Verificou-se que ser do sexo feminino, ter medo de infetar a família e amigos, a pandemia afetar negativamente a profissão, a perda de rendimentos e as novas condições de trabalho são preditores estaticamente significativos para o aumento da ansiedade $(\mathrm{p}<0,05)$. Pelo contrário, à medida que o número de anos de prática clínica aumenta, a ansiedade diminui significativamente $(\mathrm{p}=0,006)$. Conclusões: A pandemia de COVID-19 afetou negativamente os profissionais de saúde oral. As medidas de prevenção e os protocolos de controlo de infeção devem ser rigorosamente cumpridos, de modo a que haja diminuição da transmissão do vírus e, consequentemente, diminua a ansiedade, o stress e o medo sentidos por estes profissionais.

http://doi.org/10.24873/j.rpemd.2022.01.928

\section{\#072 Hábitos parafuncionais na população residente em Portugal no contexto da pandemia Covid-19}

Andreia Pires Lopes*, Maria Carlos Lopes Cardoso Real Dias Quaresma, João Manuel Mendes Caramês

Faculdade de Medicina Dentária da Universidade de Lisboa

Objetivos: Avaliar a prevalência dos hábitos parafuncionais e da ansiedade desenvolvida durante a pandemia da Covid-19 e, analisar a relação entre os mesmos e com as variáveis sociodemográficas, na população residente em Portugal. Materiais e métodos: Para a recolha de dados recorreu-se à aplicação do questionário online composta por 33 questões, dos quais encontravam-se incluídas perguntas sociodemográficas, da Escala de Ansiedade do Coronavirus e da Lista de Avaliação dos Comportamentos Orais. O mesmo encontrava-se disponível entre o mês de janeiro e março de 2021. A análise estatística incluiu a parte descritiva e inferencial. A última foi feita utilizando o teste t-student, One-Way ANOVA e correlação linear de Pearson $(\alpha=0,05)$. Resultados: A amostra foi constituída por 710 respostas válidas, sendo a maior parte constituída por mulheres. A média dos níveis de ansiedade obtida foi considerada baixa, ainda assim, foram encontrados resultados estatisticamente significativos. Quanto aos comportamentos orais, 99,9\% dos indivíduos tiveram algum tipo de comportamento oral, sendo verificada uma relação estatisticamente significativa entre o mesmo e as variáveis sociodemográficas. Foi ainda verificado, uma relação estatisticamente significativa entre a Escala de Ansiedade do Coronavirus e a maioria dos comportamentos orais, contudo esta relação variava desde fraca positiva a ínfima positiva. Conclusões: De acordo com os níveis de ansiedade e prevalência dos hábitos parafuncionais verificados na amostra concluiu-se que, apesar da correlação entre as duas escalas serem estatisticamente significativas não foram clinicamente significativas.

http://doi.org/10.24873/j.rpemd.2022.01.929

\#073 A Clorexidina na prevenção da pneumonia associada à ventilação: Uma revisão sistemática

Gustavo Manuel da Cunha e Castro*, Carla Patrícia Peixoto Babo, Maria de Lurdes Lobo Pereira

Faculdade de Medicina Dentária da Universidade do Porto

Objetivos: Estudar a eficácia da clorexidina na prevenção da Pneumonia Associada à Ventilação, considerando o seu desempenho como agente antissético integrado na rotina de higiene oral dos pacientes internados em Unidades de Cuidados Intensivos. Materiais e métodos: A revisão sistemática foi 
desenvolvida de acordo com a declaração Preferred Reporting Items for Systematic Reviews and Meta-Analyses. Foi executado e registado um protocolo de pesquisa no International Prospective Register of Systematic Reviews. O estudo foi orientado pela seguinte questão de investigação: A clorexidina, como agente antissético integrado na rotina de higiene oral dos pacientes internados em Unidades de Cuidados Intensivos, é eficaz na prevenção da Pneumonia Associada à Ventilação?. A pesquisa foi realizada nas bases de dados Cochrane CENTRAL, PubMed ${ }^{\circledR}$, Web of ScienceTM, ScienceDirect e Dentistry and Oral Sciences Sources. O processo de seleção de artigos decorreu em duas fases, de forma independente, por dois avaliadores. Os títulos e os resumos dos artigos resultantes da remoção de duplicados foram avaliados e depois foi feita a análise do texto integral dos artigos selecionados. Resultados: O processo de seleção resultou em 10 artigos, dos quais 6 ensaios clínicos randomizados, 2 estudos longitudinais, 1 estudo transversal e 1 estudo quase-experimental. Todos analisaram de forma direta a influência da clorexidina e das intervenções comparativas na incidência da Pneumonia Associada à Ventilação, sendo que dois concluíram que o colutório de clorexidina a $0,2 \%$ era eficaz na redução da incidência da Pneumonia Associada à Ventilação, um concluiu o mesmo para uma concentração de $2 \%$ de clorexidina e outro concluiu que a clorexidina apresentava menor eficácia que a intervenção comparativa, de forma estatisticamente significativa. Dos restantes 6 estudos, que obtiveram resultados sem significância estatística, quatro constataram a eficácia da clorexidina na redução da incidência de Pneumonia Associada à Ventilação e dois verificaram que a clorexidina era menos eficaz que a intervenção comparativa para esse propósito. Conclusões: A clorexidina assume-se como um agente de higiene oral potencialmente eficaz na prevenção da Pneumonia Associada à Ventilação em Unidades de Cuidados Intensivos.

http://doi.org/10.24873/j.rpemd.2022.01.930

\section{\#074 Os efeitos biológicos das resinas 3D usadas em Medicina Dentária: Revisão sistemática CrossMark}

Anabela Paula*, Inês Alexandre Neves Francisco, Madalena Prata Ribeiro, Carlos Miguel Marto, Eunice Virgínia Carrilho, Francisco Vale

Instituto de Ortodontia da Faculdade de Medicina da Universidade de Coimbra, Instituto de Clínica Integrada da Faculdade de Medicina da Universidade de Coimbra

Objetivos: Os dispositivos médico-dentários termoplásticos têm sido utilizados de forma crescente nos últimos anos após o aparecimento das tecnologias digitais. Na Ortodontia, as terapêuticas com alinhadores têm ganho maior destaque, especialmente devido às políticas agressivas de promoção desenvolvidas pela indústria. No entanto, os seus efeitos sistémicos não são conhecidos e existem poucos estudos sobre a avaliação da toxicidade sistémica destes materiais. A libertação de bisfenol A e outros monómeros residuais têm efeitos citotóxicos, genotóxicos e estrogénicos. O objetivo desta revisão sistemática é analisar sistematicamente a qualidade da evidência existente quer em estudos in vitro, quer em estudos clínicos. Materiais e métodos: A questão PICO formulada foi ' A utilização de dispositivos resinosos 3D induz efeitos citotóxicos ou alterações dos níveis de estrogénios?". A pesquisa foi realizada em várias bases de dados e segundo as normas PRISMA. Foram incluídos estudos in vitro, in vivo e clínicos. O risco de viés foi avaliado através das ferramentas da Cochrane. A avaliação da qualidade da evidência foi realizada através da ferramenta GRADE. Resultados: Um total de 236 artigos pesquisados em várias bases de dados foram inicialmente escrutinados. O risco de viés foi considerado médio a baixo. A análise da qualidade da evidência foi considerada baixa a média. Conclusões: Os efeitos citotóxicos ou nos níveis de estrogénios não pode ser confirmada com base na evidência preliminar limitada dada pelos estudos in vitro. A evidência de libertação de bisfenol A e outros monómeros dos dispositivos termoplásticos, quer nos estudos in vitro, quer nos estudos clínicos, mantêm-se ambígua. Os resultados poucos robustos da literatura atual demonstram a absoluta necessidade de mais estudos, especialmente devido às eventuais implicações sobre a fertilidade de doentes jovens, uma vez que constituem um dos maiores grupos portadores destas terapêuticas ortodônticas. http://doi.org/10.24873/j.rpemd.2022.01.931

\section{\#075 Comportamento de iões metálicos cromo} e cobalto - Coeficientes de difusão mútua

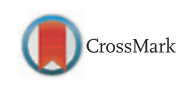

Sónia Fangaia*, Ana Cristina Ribeiro, Artur Valente, Miguel Esteso, Fernando Guerra, Pedro Nicolau

Departamento de Química da Universidade de Coimbra, Universidade Católica Santa Teresa de Jésus de Ávila - Espanha, Instituto de Implantologia e Prostodontia Faculdade de Medicina da Universidade de Coimbra

Objetivos: Estudar os coeficientes de difusão dos iões cromo e cobalto sob a forma de sais, presentes nas próteses dentárias, em meios com diferentes $\mathrm{pH}$, nomeadamente saliva artificial com $\mathrm{pH}=7,0 ; 8,3$ e 2.3 e saliva artificial fluoretada com $\mathrm{pH}=7,0$ e 2,3. Materiais e métodos: Soluções contendo cloreto de cobalto (II) hexa-hidratado (Sigma-Aldrich ${ }^{\circledR}$ ) pró-análise e cloreto de cromo (III) hexa-hidrato (Riedel-de-Haen ${ }^{\circledR}$ ) pró-análise com uma pureza (fração de massa)> 0,99 e 0,97 , respetivamente foram usados sem mais purificação. Estas soluções para as medidas dos coeficientes de difusão foram preparadas com água Millipore-Q (resistência específica $=1,82 \times 105 \Omega \mathrm{m}$, a $298,15 \mathrm{~K}$ ). Todas as soluções foram preparadas e usadas imediatamente, a 298,15 K em cada ensaio. Utilizou-se um modelo experimental pseudo-binário, baseado na técnica de dispersão de Taylor em que se faz uma injeção de um pequeno volume de cada solução, contendo o solvente (saliva artificial com $\mathrm{pH}=7,0,8,3$ e 2.3; e saliva artificial fluoretada com $\mathrm{pH}=7,0$ e 2,3) e o soluto, neste caso ião/ iões a analisar; nesse solvente em fluxo laminar através de um longo tubo capilar. Após a injeção, o pulso é disperso pela ação do gradiente de concentração e devido ao perfil parabólico de velocidades do eluente. Os resultados são medidos por um refratómetro diferencial e o tratamento matemático dos dados é baseado na 2. ${ }^{\text {a }}$ lei de Fick. Resultados: Em saliva artificial com pH=2,3, com ácido láctico, ou fluoreto de sódio, 